\title{
TUMOURS OF RENAL PELVIS
}

\author{
By I. H. GRIFFITHS, F.R.C.S. \\ From the Urological Department, Middlesex Hospital
}

At the Annual Meeting of the British Association of Urological Surgeons in June I95 I a survey was made of 23I4 renal and ureteric tumours collected from 67 Centres in the British Isles (Riches, Griffiths and Thackray (195I)).

In this series 315 ( 12.5 per cent.) were tumours of the renal pelvis, an incidence of about $I$ in I 200 of malignant tumours admitted to General Hospitals.

This long series of an interesting, though comparatively rare, condition presented an opportunity to study the clinical and pathological behaviour and to determine what factors influence the prognosis. Many of the conclusions of this series serve only to confirm those of J. B. Macalpine (1947) to whom credit is given for much valuable and original work on this subject.

\section{Aetiology}

Like most other tumours of the urinary tract, very little is known of the aetiology. A most interesting fact arising from the collective report was that no cases of bilateral papillary tumours of the pelvis were recorded. This does suggest that the noxious agent responsible for tumour production is not excreted in the urine. In the case of papillary tumours of the pelvis it is uncommon to find any local condition which may be presumed to be a cause. The urine is usually found to be sterile, and stone, which was recorded as being present in association with papillary tumours in only 5 per cent., probably develops as a result of tumour obstruction.

In the aetiology of squamous-cell carcinoma chronic inflammation undoubtedly plays an important part. Prolonged continuous renal infection is a common finding and often gives rise to squamous metaplasia, although leukoplakia, a precarcinomatous condition, is not often observed. Associated calculous disease of the kidney was found in approximately a third of the cases, a much lower incidence than is recorded by some authorities but high enough to be significant as a predisposing cause of epitheliomata of the pelvis.

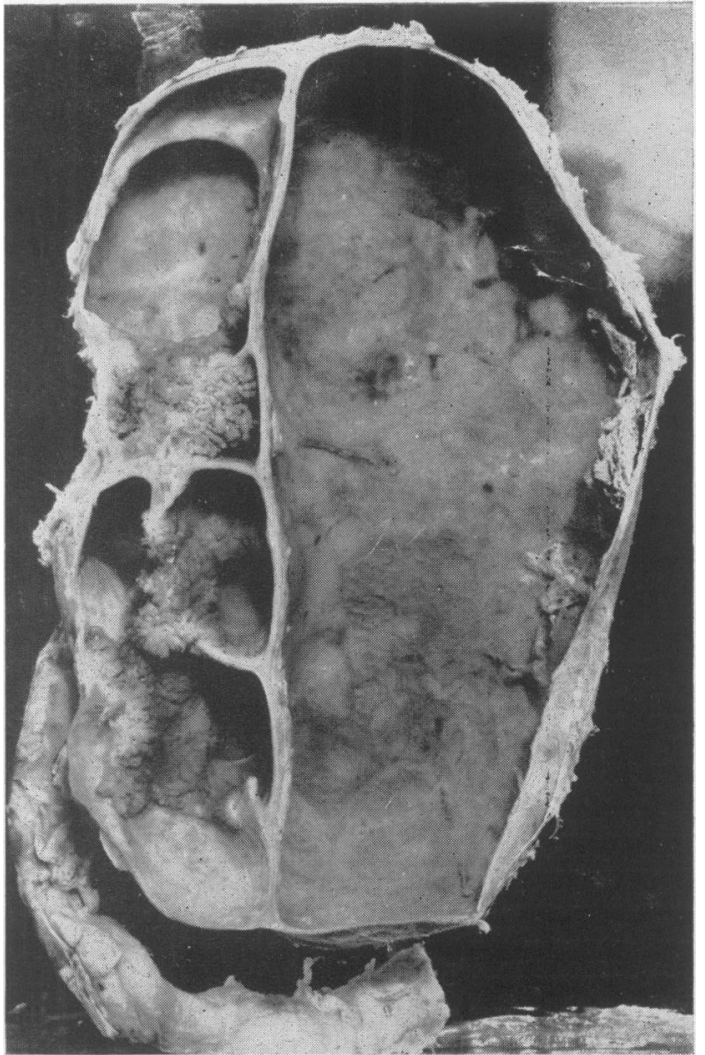

FIG. I.-Obstruction by simple papilliferous tumours of the renal pelvis causing gross hydronephrosis with destruction of the renal parenchyma.

\section{Pathology}

Transitional-cell and squamous-cell tumours arising within the renal pelvis are similar in appearance and structure to those developing within the urinary bladder, but the prognosis of the renal tumours is distinctly worse than comparable tumours of the bladder. This gloomy outlook for renal growth is, in all probability, due to the thin 
walled pelvis being penetrated more rapidly by malignant invasion.

Elaborate classification of pelvic tumours leads to confusion and a simple grouping into three primary types has much greater practical value:

(a) Transitional cell papilloma.

(b) Transitional cell carcinoma.

(c) Squamous cell carcinoma.

\section{Transitional cell Papilloma}

Simple papillomata account for about 25 per cent. of tumours of the renal pelvis and are more often multiple than single. A single tumour is occasionally found near the pelvi-ureteric junction causing obstruction, pain and haematuria early in its development. In less fortunate cases these tumours may arise within the pelvis or a calyx and develop silently into large and multiple papillary tumours before revealing themselves (Fig. I).

The simple nature of these tumours is doubted by many pathologists, and with good reason, since simple papillomata of the pelvis may be found in association with papillary carcinoma in the ureter or bladder. However, from the clinical aspect, it is wise to make a distinction between benign and malignant tumours since the prognosis in each case is quite different.

\section{Transitional cell Carcinoma}

These tumours constitute about 50 per cent. of growths of the kidney pelvis. They are usually bulkier tumours than the simple papillomata and are more apt to give rise to hydronephrosis or hydrocalycosis, and in each cases the distended organ may be found indurated by multiple tumours studding the wall. The villi may be long and the pedicles narrow, but, as malignancy advances, the villi become short and stumpy and the pedicles become wider, giving rise to a nodular type of growth showing a higher degree of malignancy and greater invasion of the pelvic wall.

Squamous metaplasia to a varying degree occurs frequently, but tumours showing this transition should not be regarded as epitheliomata which show a significant difference in mode of spread and in prognosis.

\section{Squamous cell Carcinoma}

Epithelioma was recorded in 22 per cent. of cases of pelvic tumours. It is commonly a flat infiltrating tumour but may be a bulky growth involving the pelvis and renal parenchyma (Fig. 2).

\section{Ureteric and Bladder Involvement}

An interesting feature of papillary tumours of the renal pelvis is the secondary development of similar tumours in the ureter and bladder. Such extension was found in as many as 43 per cent. in

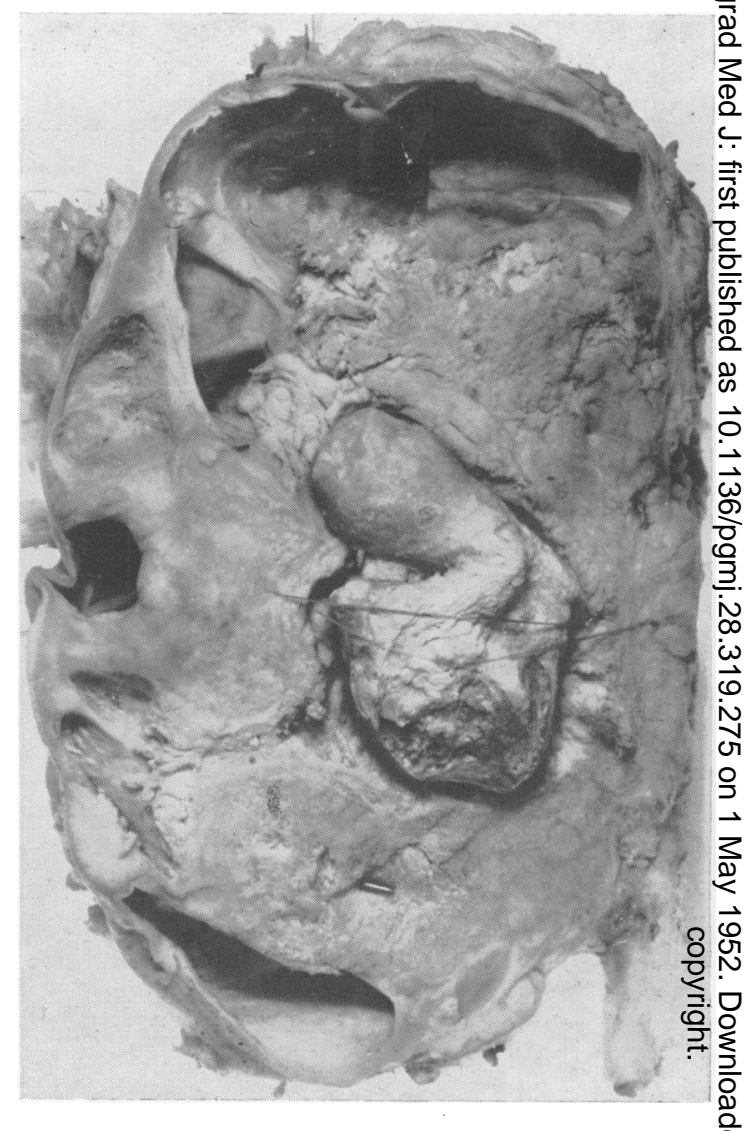

FIG. 2.-Bulky squamous cell tumour of renal pelvis $\stackrel{\odot}{\circ}$ involving the parenchyma. Note the large pelvic $\vec{F}$ calculus embedded in growth.

transitional cell carcinoma and in about 44 per cent. of simple papillomata.

In squamous cell carcinomata the involvement of ureter was seen in about 13 per cent., the mode $\frac{3}{3}$ of spread being in most cases by direct continuity.

\section{Metastases}

Malignant extension to distant organs occurs음 with equal frequency in transitional cell and $>$ squamous cell carcinomata of the pelvis. The lung and liver are the most common organs affec-N ted, the regional lymph nodes and bones are less frequently involved. The sites of secondary deposits in bone in order of frequency are thew vertebrae, ribs and femora. In almost $5^{\circ}$ pero cent. of cases where metastases occurred singlec sites were recorded, a feature typical of paren- $\$$ chymal carcinoma of the kidney.

The delayed appearance of ureteric stump $\frac{0}{0}$ tumours following nephrectomy occurred with $\stackrel{\vec{\Phi}}{\circ}$ remarkable frequency and this knowledge must $\stackrel{?}{\square}$ have important bearing upon the plan of treatment $\stackrel{\mathbb{}}{\sigma}$ 
of this renal disease. In 8 per cent. of simple papillary tumours of the pelvis the ureteric stump was involved, and for transitional cell carcinoma of the pelvis the incidence was 9 per cent., whilst for squamous call carcinoma it was only 3.6 per cent. Thus, it appeared that the papillary tumours showed a greater tendency to delayed appearance, and in the majority of cases this took place vithin a year.

\section{Theories of Tumour Dissemination}

The secondary appearance of tumours in the subpelvic regions of the urinary tract is a pathological feature which has many theories to account for it. Two most attractive possibilities are $(a)$ implantation of seedlings and $(b)$ origin from multicentric foci in the urinary tract.

In the former theory, papillomatous material from the primary lesion in the pelvis is alleged to be transferred down the ureter in the urinary stream and becomes held up at some constricted part, most commonly at the juxta-vesical segment, and, after a period of close contact with the ureteral mucosa, develops a blood supply from it. This would account for multiple and unilateral distribution of the disease but does not explain the association of malignant tumours in the lower urinary tract with simple tumours of the pelvis. A most enigmatic feature of the disease is the occurrence of ureteric stump tumours, for in more than 80 per cent. of these cases the tumours were revealed within one year after nephrectomy. It is suggested that in such cases ureteric implants were present at the time of nephrectomy but went unobserved by the surgeon.

The origin of tumours from multicentric foci is a conception that considers the epithelium of the urinary tract to have an inherent propensity to tumour formation. This property is thought to be a regional feature which explains the unilateral development of multiple tumours. That these tumours show differences in character is due to different degrees of advancement. Thus, it becomes possible to have simple and malignant papilliferous tumours in close or distant association with each other.

\section{Age, Sex and Side Incidence}

More than 80 per cent. of the more common tumours of the renal pelvis occur in the 5 th, 6 th and $7^{\text {th }}$ decades. An exceptional case in the B.A.U.S. series was of a transitional cell carcinoma developing in a child of 9 .

Males are affected three to four times more often than females except in the case of squamous cell tumours, where male incidence only slightly exceeds female. There appears to be no preference for side, for in all tumours of the renal pelvis the incidence of right and left is almost identical.

\section{Symptoms and Signs}

Pain, Haematuria and Tumour is a symptomatic triad found in varying proportions and severity in all tumours of the urinary tract. Haematuria is the leading symptom and occurs in about 90 per cent. oi cases of transitional cell carcinoma and simple papilloma, whilst pain is present in about 45 per cent. The high incidence of pain in these cases must be due to associated urinary infection, passage of blood clots, and distension of the renal pelvis. In cases of squamous cell carcinoma the incidence is about 60 per cent. The frequent association of stone with renal epitheliomata must be mainly responsible for the increased incidence of pain.

\section{Tumour}

Tumours of the renal pelvis may cause gross dilatation of the pelvis and calyceal system, but they are seldom clinically palpable. In contrast to tumours of the renal parenchyma, which are palpable in 60 per cent. of cases, only 2 per cent. of renal pelvis tumours are revealed on abdominal examination. Dilatation of the ureter, or the

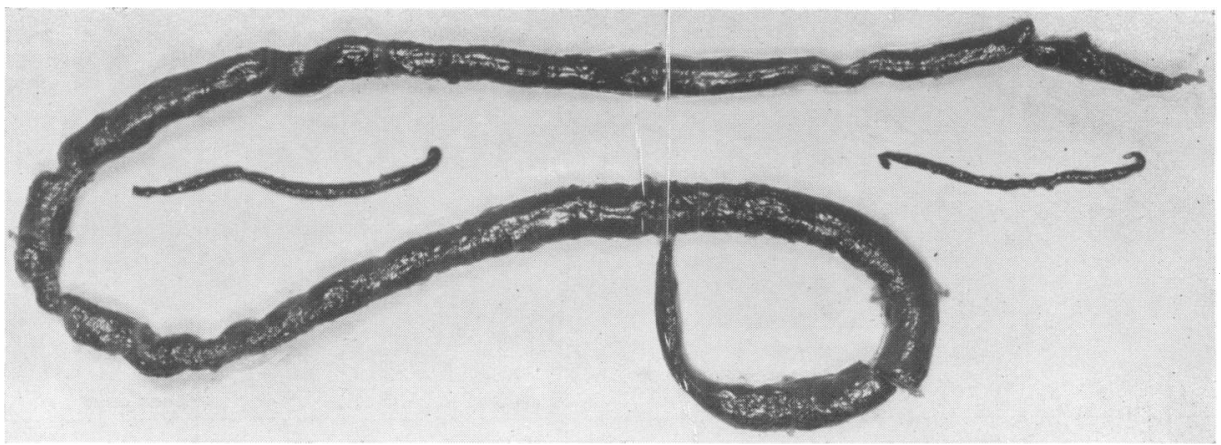

FIG. 3.-A blood cast of ureter from a case of papilliferous tumour of the renal pelvis. 
pelvis, or the calyceal system may occur depending on the site of tumour development, but it is not usually appreciated before radiological examination.

\section{Investigations}

Haematuria demands an early and full urological investigation. The urine must be examined to establish the presence of blood, which sometimes may be only microscopic and at other times may be profuse and clotted. Vermiform blood clot, a cast of the ureter, is a reliable sign of profuse haemorrhage from the upper part of the urinary tract (Fig. 3).

In this disease of the upper urinary tract diagnosis is often difficult and one can sympathize with the clinician who fails to detect a tumour too small to be seen radiographically. But, so frequently, transient episodes of haematuria are spaced by long periods of silence during which time both doctor and patient are willing to forget the ominous and colourful warning so providently arranged by nature.

\section{Cystoscopy}

Cystoscopy has the greatest value in the presence of haematuria, when the source of haemorrhage may be determined; if it is of renal origin, a bloody efflux from a ureteric orifice will reveal the offending side. If cystoscopy during a quiescent period does not produce a diagnosis with certainty then it is wiser to re-examine during the period of haemorrhage than to make a doubtful diagnosis of such a condition as varicose veins of the bladder.
Papilliferous growths may be seen near or evenڤึ within a ureteric orifice. In the case from which the specimen shown in Fig. 4 was removed, theo fronds of a ureteric tumour were seen protruding. through the ureteric orific and occasionally would retract from view to reappear with the next urinary efflux.

\section{I.V.P.}

The radiographic picture depends entirely on the stage of advancement of the growth and on ${ }^{\circ}$ the site and degree of obstruction. There is noo one pyelogram that is typical of a pelvic growth, $\vec{\overrightarrow{ }}$ but almost all"will fall under one of the following headings :

(a) The pelvic tumour may be too small to cast 3 an X-ray shadow, and a final diagnosis is madei after nephrectomy for haemorrhage.

(b) Obstruction by growth may cause dilatation $\frac{\omega}{\bullet}$ of the ureter, pelvis or calyces, and a filling defectro may be seen at the site of obstruction or withinor the dilated part of the urinary tract. Occasionally, the presence of a villous tumour can be appreciatedby the mottled appearance of the filling defect? (Fig. 5).

(c) A bizarre appearance may be seen reminis- $\overrightarrow{0}$ cent of pyelograms of some large cortical tumouss N and is due to the small amount of contrast medium? that is able to penetrate between the fronds of bulky and tightly compressed pelvic tumour.

(d) The kidney may be non-functioning as in the more advanced tumours which may involve $\frac{\circ}{\mathbb{D}}$ the whole of the parenchyma, or where tumour

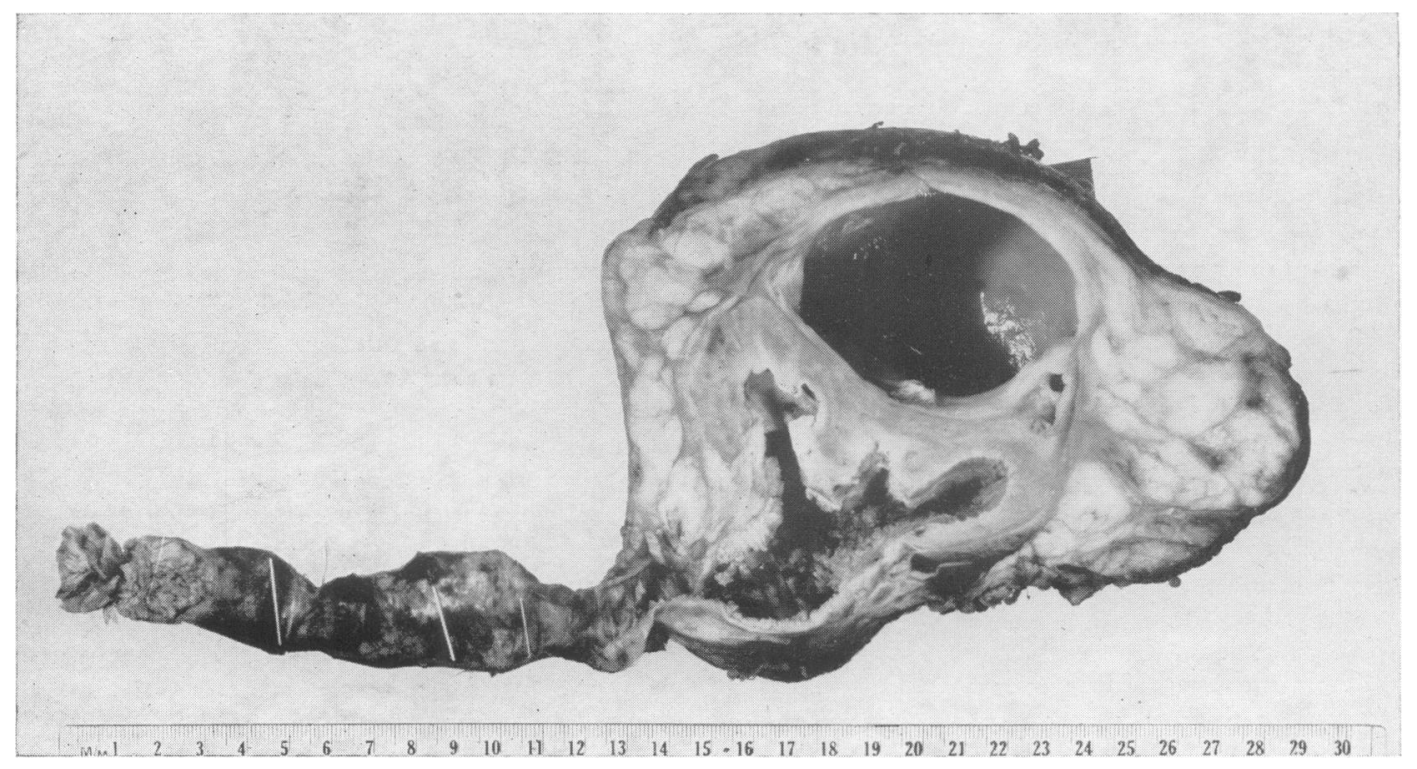

FIG. 4.-Papilliferous carcinoma of the pelvis with involvement of the ureter throughout its length. The long fronds of tumour at the lower end were seen on cystoscopy. 


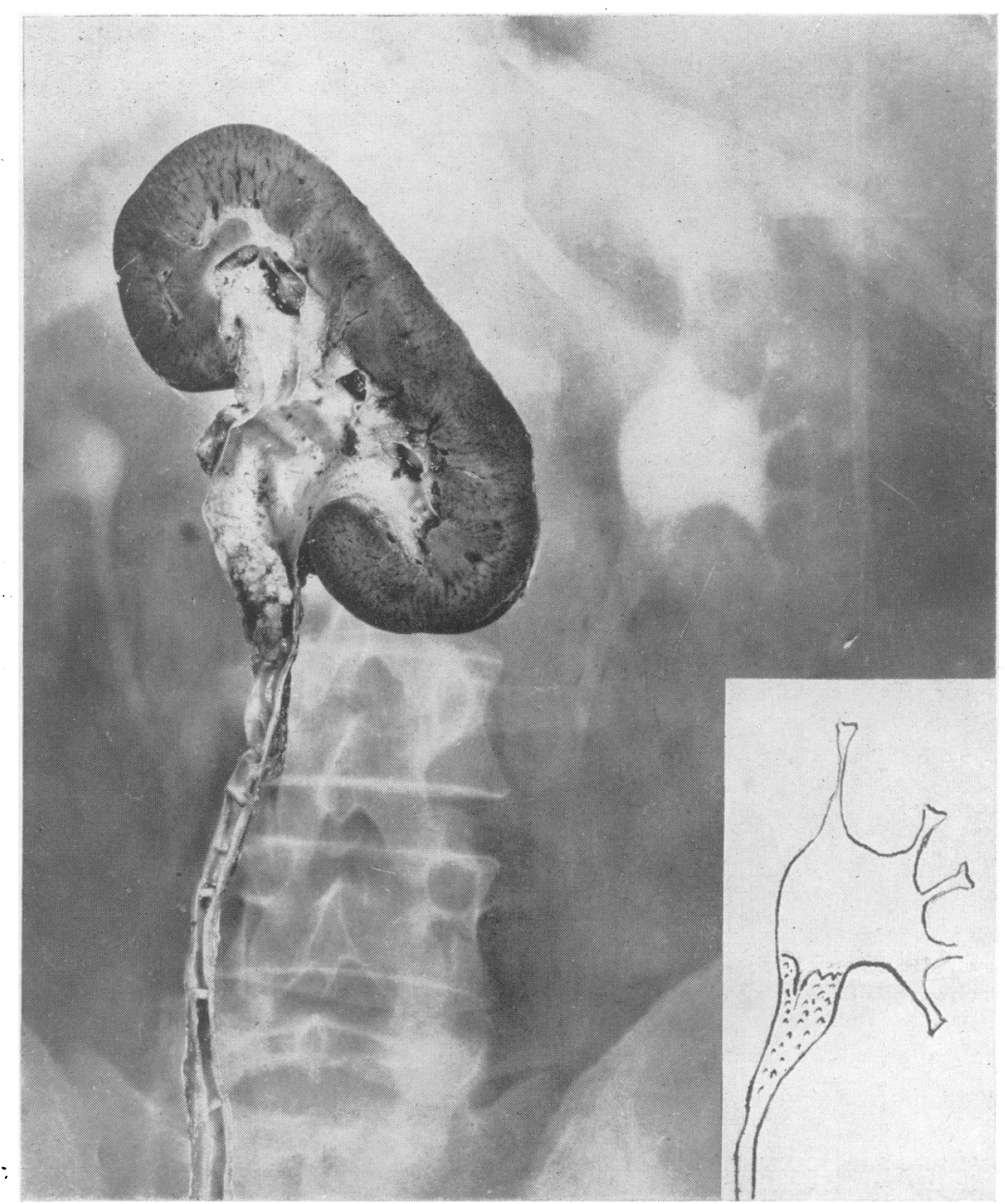

FIG. 5.-A specimen of a villous transitional cell carcinoma of the pelvi-ureteric junction superimposed on the pyelogram. Note the filling defect in the pelvis as shown in the inset diagram. Mottling in the filling defect can be seen distinctly in the original $\mathrm{X}$-ray and is diagnostic of a papillary tumour.

obstruction of the urinary tract has become complete.

When nephrectomy is under consideration an I.V.P. is of supreme importance in determining the function of the opposite kidney.

\section{Retrograde Pyelography and Ureterography}

The main indications for retrograde pyelography are:

(a) Where absent excretion of one kidney is found on I:V.P.

(b) Where definition of the I.V.P. is not good and the result inconclusive.

It is a wise routine procedure to confirm the findings of the I.V.P. Confusion arises when the space occupying condition of the pelvis is an air bubble or a blood clot (Fig. 6). The air bubble is always perfectly round and is usually associated with other bubbles in the ureter. But, if either of these conditions is questioned, then repeat of the retrograde pyelogram should be performed after first washing out the pelvis with saline and taking precautions against the reintroduction of air with the contrast medium.

The pyelographic distinction between pelvic and cortical tumours is important in the consideration of treatment. The main differences have been generalized by Riches (I95I), who states that cortical tumours produce their main effects on the calyces, whilst pelvic tumours, whether papillary or squamous, affect the pelvis.

Passage of a catheter to the kidney may not be possible due to obstruction in the course of the ureter. A ureterogram with the catheter intro- 


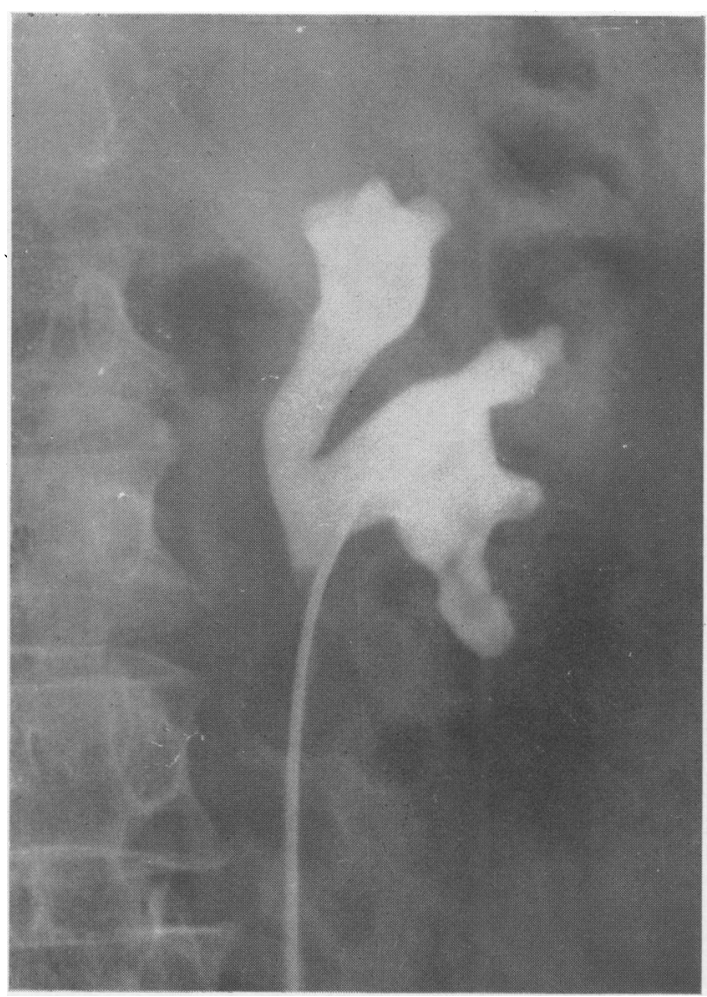

FIG. 6.-Filling defect seen in the lower calyx proved to be a blood clot as the result of haemorrhage from the upper calyx which once contained a calculus.

duced as far as it will go may occasionally bring to view a filling defect with dilatation of ureter above. This investigation is not as important as others previously mentioned, but it may demonstrate the presence of a tumour, whether primary or seedling, for which the treatment is identical.

\section{Treatment}

The ideal treatment for all tumours of the renal pelvis is nephro-ureterectomy. Exploration of the kidney by nephrotomy or pyelotomy must be condemned since tumour spill into the wound of approach is almost unavoidable.

Confirmation of radiological diagnosis can sometimes be made by palpation of the exposed kidney. A papillary tumour within the pelvis is commonly described as giving a sensation like a wad of wet cotton wool.

The kidney is exposed through a lumbar incision and its pedicle ligated and transected. Without sectioning the ureter, the kidney is exteriorized and the lumber wound closed. A second incision is made in the iliac fossa, parallel to the inguinal ligament, and the lower end of the ureter exposed extra peritoneally. There is diversity of opinion about the point of section of the ureter. Transec $z$ tion of the ureter flush with the bladder followed by transurethral diathermy of the intra-muraFureter is an accepted method of treatment, but $i$ carries with it a great risk of tumour spill into the wound of approach. A more formidable operation is the removal of a disc of bladder around the ureteric orifice. This has the merit of being complete removal of ureter with less danger of tumour spill through accidental section throughis growth, but nevertheless, it does not obviate spill $\vec{b}$ of urine which may contain fragments of growth.-

The general condition of the patient naturally, govern; the choice of methods used and, if the simultaneous removal of ureter and kidney is not justified, a compromise can be reached by staging. the operation. The kidney and upper part ofo ureter is excised as a first stage and, when the condition of the patient permits, the stump of ureter is removed.

\section{Prognosis}

The prognosis depends on the degree of malig nancy of the growth and the extent to which it has spread outside the confines of the urinary tracto before its removal.

Transitional cell carcinomata are graded intor high degree of malignancy when the villi are shore and stumpy, the cells vary in size and depth of staining, and mitosis easily found; and into a lowo degree when the villi are long and slender, anco the cells regular in size and pattern (Figs. 7 and 8$) \stackrel{\varrho}{\rightleftarrows}$

The influence of ureteric and bladder involvemene on survival is not striking. Tumours of low grade malignancy with extension to the ureter an bladder do much better than high grade tumours apparently confined to the renal pelvis. It happens that the majority of papillary tumours associated with ureteric and bladder extension are of high?. grade malignancy, but, in any case the prognosis is influenced almost entirely by grade of malig nancy and not by involvement of subpelvic regionso of the urinary tract.

Epitheliomata are the most highly malignane tumours of the pelvis but they show surprising restraint from distant extension to the ureter ando bladder.

\section{Survival}

Simple Papilloma.-In the B.A.U.S. series about 90 per cent. survive three years and 50 per cent $\$$ five years. The small number of cases followed up for to years make survival figures unreliable.

Transitional cell Carcinoma.-The survival tables of operable cases of transitional cell carcinomata of the pelvis showed no marked difference betweer? 


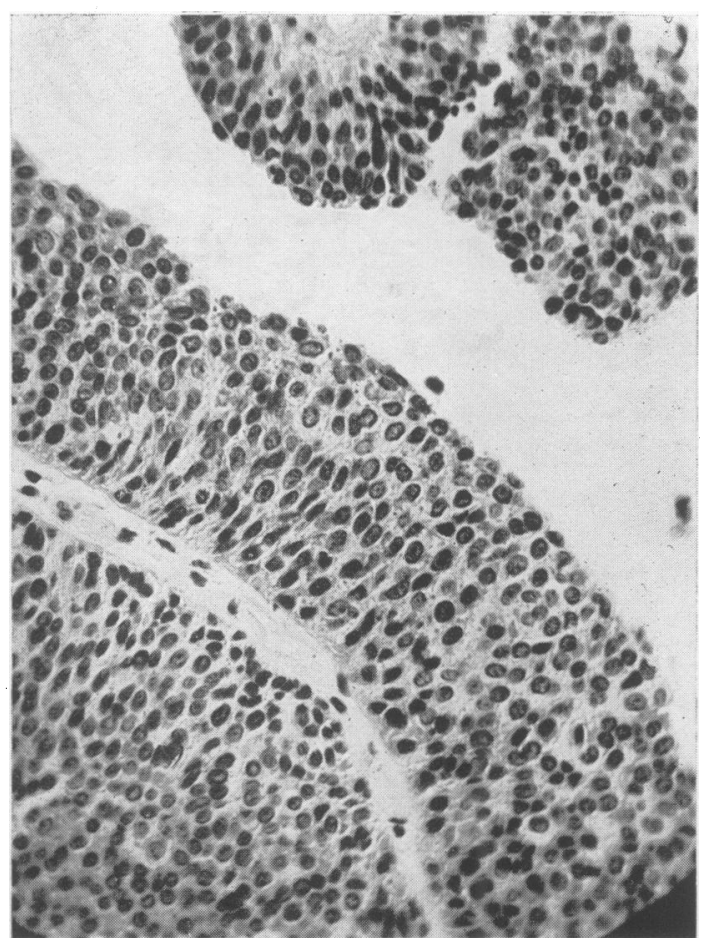

Fig. 7.-Transitional cell carcinoma of low degree of malignancy. The cells are regular in size and pattern, and only occasional mitotic figures are seen.

those cases involving and not involving ureter or bladder, and the figure combining these two series is probably more accurate. Nearly 70 per cent. survive one year and nearly $5^{\circ}$ per cent. survive three years. Only 25 per cent. are alive in ro years.

Squamous cell Carcinoma.-In this rare but exceptionally malignant condition, only a third of the cases survive one year and there were no survivals beyond three years.

I am indebted to the Honorary Editor of the British Fournal of Urology for his kind permission

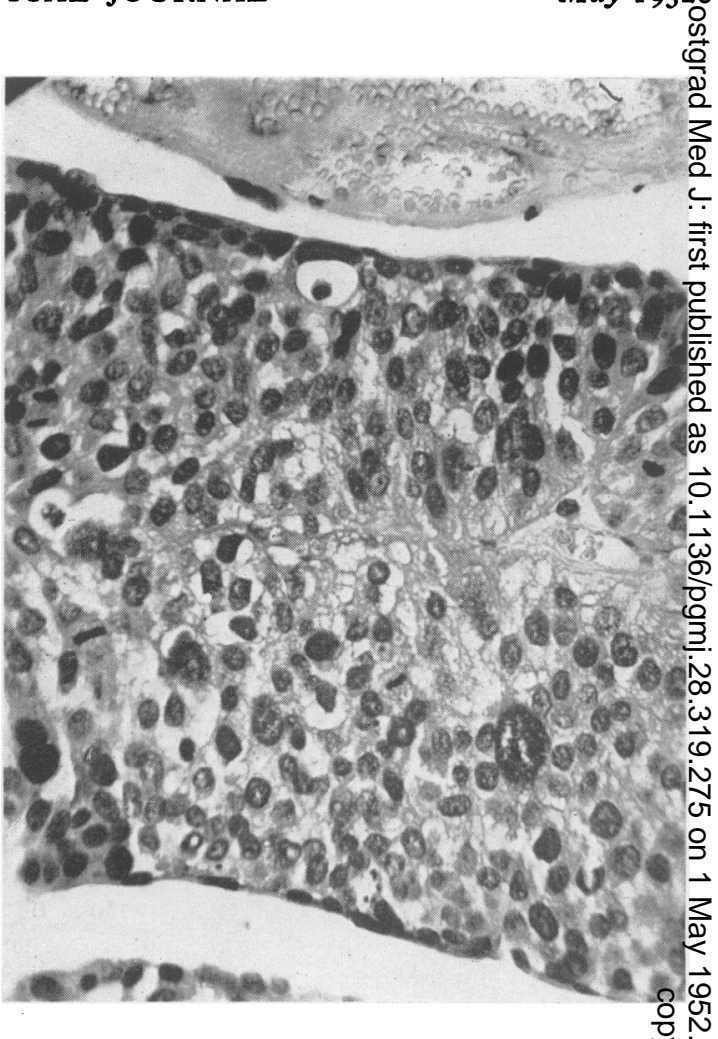

FIG. 8.-Transitional carcinoma of high degree fi malignancy and the same magnification as in Fig. The cells are large and vary in depth of staining, the pattern is irregular and mitosis is frequent. $\times$

$\stackrel{2}{\frac{1}{8}}$

to publish this summary. A full report may be $\overrightarrow{7}$ seen in the British Fournal of Urology, December 195I. I am grateful to Mr. E. W. Riches for his? advice and his permission to use his case noteso. and illustrations, to Dr. A. C. Thackray for the photomicrographs and to Mr. Turney, F.R.P.S., of the Middlesex Hospital Photographic Dept.

\section{BIBLIOGRAPHY}

MACALPINE, J. B. (1947), Brit. F. Surg., 35, I 13. RICHES, E. W., GRIFFITHS, I. H., and THACKRAY, A. C.O (1951), Brit.' F. Urol., 23, 297.

\section{EDITORIAL-Continued from page 267}

centres. Foetal tissues have been used but these die in the same manner as adult tissues. Attempts have been made to modify the donor tissue either chemically or physically but these have failed. Desensitising the donor tissue by growing it in a tissue culture medium of the host's serum has given unconvincing results. In a like manner, modification of the host's reaction has been carried out by inhibiting the action of the reticulo- $\frac{\omega}{\sigma}$ endothelial system, either by total body irradiation, cortisone, A.C.T.H. or nitrogen mustard, all of $\frac{C}{\Phi}$ which work but only temporarily. This problem $\stackrel{?}{+}$ awaits a solution but work is proceeding in many ${ }_{0}^{0}$ centres and the future may provide the answer. 